\title{
Novel crystal structures for Lithium-Silicon alloy predicted by minima hopping method
}

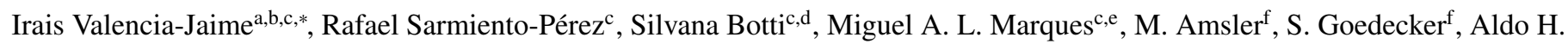 \\ Romero $^{\mathrm{a}, \mathrm{b}}$
}

\begin{abstract}
We present an ab initio study of the phase diagram of the Li-Si binary system using the minima hopping method for global structural prediction. By varying the chemical composition we obtained the theoretical convex hull, whose vertices are constituted by three stoichiometries, two of them have not been reported previously. Additionally, we find five more unreported meta-stable structures, which are slightly higher in energy with respect to the convex hull line. We further characterize all these obtained phases by calculating their electronic, mechanical, and dynamical properties. Finally, and as this class of systems is viewed with interest for possible anodes in lithium batteries, we also report the potential-composition curve and compare it with available experimental measurements.
\end{abstract}

Keywords: Minima Hopping Method, Li-Si alloys, Li-ion battery, Density functional theory PACS: 31.15.A-, 82.47.Aa, 61.05.-a

\section{Introduction}

In the past few years, the development of lithium-ion batteries (LIBs) with higher energy capacity has been the focus of many research projects, mainly due to the several potential applications for portable electronic devices and electric vehicles. Lithium-graphite compounds are the most commonly used materials as negative electrodes in LIBs due to their low price, low working voltage, and good energy density. In these compounds, $\mathrm{Li}$ atoms are intercalated between the graphite sheets during the charging cycle and removed during discharge with a minimal structural change and a $10 \%$ variation in volume [1].

Recently, silicon has been studied as a potential negative electrode in LIBs batteries, primarily because this material has the highest known theoretical storage capacity. The predicted charge capacity is close to $3500 \mathrm{mAh} \mathrm{g}^{-1}$, comparable to graphite, $371 \mathrm{mAh} \mathrm{g}^{-1}$ [2]. However, the lithiation of silicon has been associated with a volume expansion of $300 \%$ [3, 4], generating large mechanical strain, and resulting in capacity loss and very limited cycle life [5]. Therefore, it is important to have a clear view of the different crystalline structures of LiSi involved in the process, to understand the causes of this behavior and propose possible solutions.

The stable experimentally reported stoichiometries are $\mathrm{LiSi}$ (I41/a, 16 atoms per unit cell), $\mathrm{Li}_{12} \mathrm{Si}_{7}$ (Pnma, 152 atoms),

\footnotetext{
${ }^{*}$ Corresponding author

Email address: irvalenciajaime@mail . wvu.edu (Irais Valencia-Jaime)
}

$\mathrm{Li}_{7} \mathrm{Si}_{3}$ ( $P 3_{2} 12,60$ atoms), $\mathrm{Li}_{13} \mathrm{Si}_{4}$ (Pbam, 34 atoms), $\mathrm{Li}_{22} \mathrm{Si}_{5}$ (F23, 108 atoms), and $\mathrm{Li}_{21} \mathrm{Si}_{5}$ ( $F \overline{4} m 3,104$ atoms)[6, 7, 8]. Additionally, a meta-stable crystalline phase of $\mathrm{Li}_{15} \mathrm{Si} 4$ (I $\overline{4} 3 \mathrm{~d}, 38$ atoms per unit cell) has been recently reported [9]. This structure does not appear in the normal equilibrium Li-Si phase diagram $[10,11]$, suggesting that it is possible to obtain novel meta-stable structures under extreme conditions.

From the theoretical side, several methods have recently been used to explore the most stable crystal structures as a function of the stoichiometry, such as genetic algorithms [12] and random search [13]. The crystal structures that were obtained by numerical simulations allowed the construction of theoretical phase diagrams for Li-Si alloys. In particular, Tipton et. al. [12] predicted a new $\mathrm{R} \overline{3} m$ phase of $\mathrm{Li}_{5} \mathrm{Si}_{2}$ using density functional theory (DFT) coupled with genetic algorithms. They reported that a supercell obtained from this structure with partial site occupations can represent the $\mathrm{Li}_{7} \mathrm{Si}_{3}$ structure. Moreover, they found three other unreported stoichiometries $\left(\mathrm{Li}_{4} \mathrm{Si}\right.$, $\mathrm{Li}_{7} \mathrm{Si}_{2}$ and $\mathrm{Li}_{9} \mathrm{Si}_{3}$ ) for which they only reported the dynamical stability. Chevrier et. al. [1] demonstrated that $\mathrm{Li}_{21} \mathrm{Si}_{5}$ [8] is chemically more stable than $\mathrm{Li}_{22} \mathrm{Si}_{5}$. Zeilinger et. al. [14] suggested that $\mathrm{Li}_{17} \mathrm{Si}_{4}$ is the correct stoichiometry between $\mathrm{Li}_{22} \mathrm{Si}_{5}$ and $\mathrm{Li}_{21} \mathrm{Si}_{5}$ at high temperature. Morris et.al. [13] used ab initio random structural search to discover new crystalline phases of $\mathrm{LiSi}$ and $\mathrm{Li}_{8} \mathrm{Si}_{3}$. They found several new phases of LiSi with space groups $R \overline{3}, P 4 / m m m, P \overline{1}, P \overline{3} m 1, P 2 / m$ and $I 4_{1} / a m d$, which are only $0.05,0.07,0.07,0.07,0.08$ and $0.11 \mathrm{eV}$ per formula unit higher in energy than the ground state structure. For 
$\mathrm{Li}_{17} \mathrm{Si}_{4}$ they reported a phase with $F \overline{4} 3 m$ symmetry, for $\mathrm{Li}_{8} \mathrm{Si}_{3}$ a $R \overline{3} m$ structure and for $\mathrm{Li}_{13} \mathrm{Si}_{5}$ a $P \overline{3} m 1$ structure. Many of the previous theoretical investigations performed an elastic and thermal stability analysis of the predicted structures, in addition to the calculation of the formation enthalpy.

In this work, we use the minima hopping method (MHM) proposed by Goedecker $[15,16]$ to investigate the binary phase diagram of $\mathrm{Li}-\mathrm{Si}$ at zero pressure. The article is organized as follows. In section 2, we describe the computational details of our calculations. Section 3 discusses the crystal structures obtained for all the different compositions, with a particular focus on those on the convex hull or very close to it. After selecting the lowest enthalpy structures, we perform an electronic, mechanical and dynamical characterization to describe general thermodynamic properties. We end section 3 by discussing the potential-composition curve of Li-Si binaries as a function of Li content. Section 4 summarizes our work.

\section{Computational details}

Different numerical approaches can be used nowadays to sample the potential energy surface in order to find the global energy minimum. In this work, we use the minima hopping method (MHM) for global structural prediction. This method employs an efficient dynamical algorithm to explore the energy landscape by using temperature as a key variable to overcome the potential barriers and reach lower minima. When coupled with $a b$ initio density functional theory (DFT), this method is capable of predicting stable and meta-stable crystal structures at a given pressure, from the sole knowledge of the chemical composition of the system. A series of consecutive short molecular dynamics (MD) simulations are used to escape from a local minimum by overcoming an energy barrier. We perform NPT MD simulations with a time interval of $700 \mathrm{fs}$ ( $3.5 \mathrm{fs}$ time step) and an initial temperature of $200 \mathrm{~K}$, which is potentially increased with a factor or 1.2 according to the algorithm. It is this temperature change the one responsible for the necessary energy to overcome energy barriers. After the MD simulation, local geometry relaxation is performed to identify the configuration corresponding to the new energy valley bottom. The efficiency of the escape step is improved by choosing the initial velocities of molecular dynamics trajectories approximately along of soft mode directions [15].

For the structural search calculations presented in this paper, we used unit cells containing up to 16 atoms and considering all possible stoichiometries under this restriction. The evaluation of energy and forces required for MHM were obtained from DFT as implemented in the Vienna Ab initio Simulation Package (VASP version 5.3.3) [17, 18] The projected augmented wave (PAW) method was used to describe valence and core electrons [19]. In this case the electronic configurations taken into account in the PAW pseudo-potential were: five valence electrons for $\mathrm{Li}\left(1 \mathrm{~s}^{2} 2 \mathrm{~s}^{2} 2 \mathrm{p}^{1}\right)$ and four for $\mathrm{Si}\left(3 \mathrm{~s}^{2} 3 \mathrm{p}^{2}\right)$. To approximate the exchange-correlation functional of DFT, we used the Perdew-Burke-Ernzerhof (PBE) [20] generalized gradient approximation. We used a plane wave cutoff of $550 \mathrm{eV}$, and a
Monkhorst-pack [21] method (Gamma-center $k$-grid for hexagonal and trigonal structures were used). Brillouin zone sampling grid was converged to guarantee a numerical accuracy of the total energy to less than $2 \mathrm{meV} /$ atom. In order to ensure the dynamical stability of the identified crystal structures, we calculated their phonon dispersion. The software PHONOPY [22] was used to obtain the force constants from VASP with a $2 \times 2 \times 2$ supercell. Elastic constants which converged to less than $1 \mathrm{GPa}$ were calculated from strain-stress relations as implemented in VASP. For the calculations of the elastic and electronic properties, k-point grids of size $12 \times 12 \times 12$ were used.

An important observable related to lithium batteries is the electric potential profile. The experimentally reported profiles show the open circuit voltage of a battery as a function of the anode lithium content. The average cell potential can be calculated under the assumption of a two-phase reaction from reactant to product $[6,23,24]$.

$$
\mathrm{Li}_{x} \mathrm{Si}+(y-x) \mathrm{Li} \rightarrow \mathrm{Li}_{y} \mathrm{Si},
$$

where the average voltage is defined as

$$
\text { Voltage }=-\left\{\frac{G_{\mathrm{Li}_{y} \mathrm{Si}}-G_{\mathrm{Li}_{x} \mathrm{Si}}}{y-x}-G\left(\mathrm{Li}_{\text {metal }}\right)\right\}
$$

with the Gibbs free energy $G$ defined as

$$
G=E+p V-T S
$$

Where $\mathrm{E}$ is energy, $\mathrm{V}$ is volume, $\mathrm{p}$ is pressure, $\mathrm{T}$ is temperature, and $\mathrm{S}$ is entropy. In this case, the $\mathrm{pV}$ contribution to $\mathrm{G}$ is of the order of $10 \mu \mathrm{eV}$ per $\mathrm{Li}$ at atmospheric pressure [25]. The TS contribution to the voltage is assumed to be constant and estimated to be on the order of $10 \mathrm{meV}[25,26]$. Therefore, the average potential of the electrode can be derived from the simple equation:

$$
\text { Voltage }=E(\mathrm{Li})-\frac{E\left(\mathrm{Li}_{y} \mathrm{Si}\right)-E\left(\mathrm{Li}_{x} \mathrm{Si}\right)}{y-x}
$$

Here, we have calculated total energies (or enthalpies) by means of electronic structure calculations within DFT.

\section{Results and Discussion}

We studied sixty different stoichiometries of the Li-Si system, starting from pure silicon up to pure lithium. For each stoichiometry, a structural search was performed as described in section 2. Then, the lowest energy structures were identified and selected for structural re-optimization with a tighter stress and force convergence criteria.

Combining the calculated formation energies for all different stoichiometries, we have calculated the theoretical convex hull as depicted in Fig. 1. The thermodynamically stable structures are indicated with (black) circles and define the vertices of the convex hull (black line). Low-lying local minima are represented by (magenta) crosses. Additionally, we also construct the convex hull (red dashed line) associated with the experimental structures. These structures were available through 


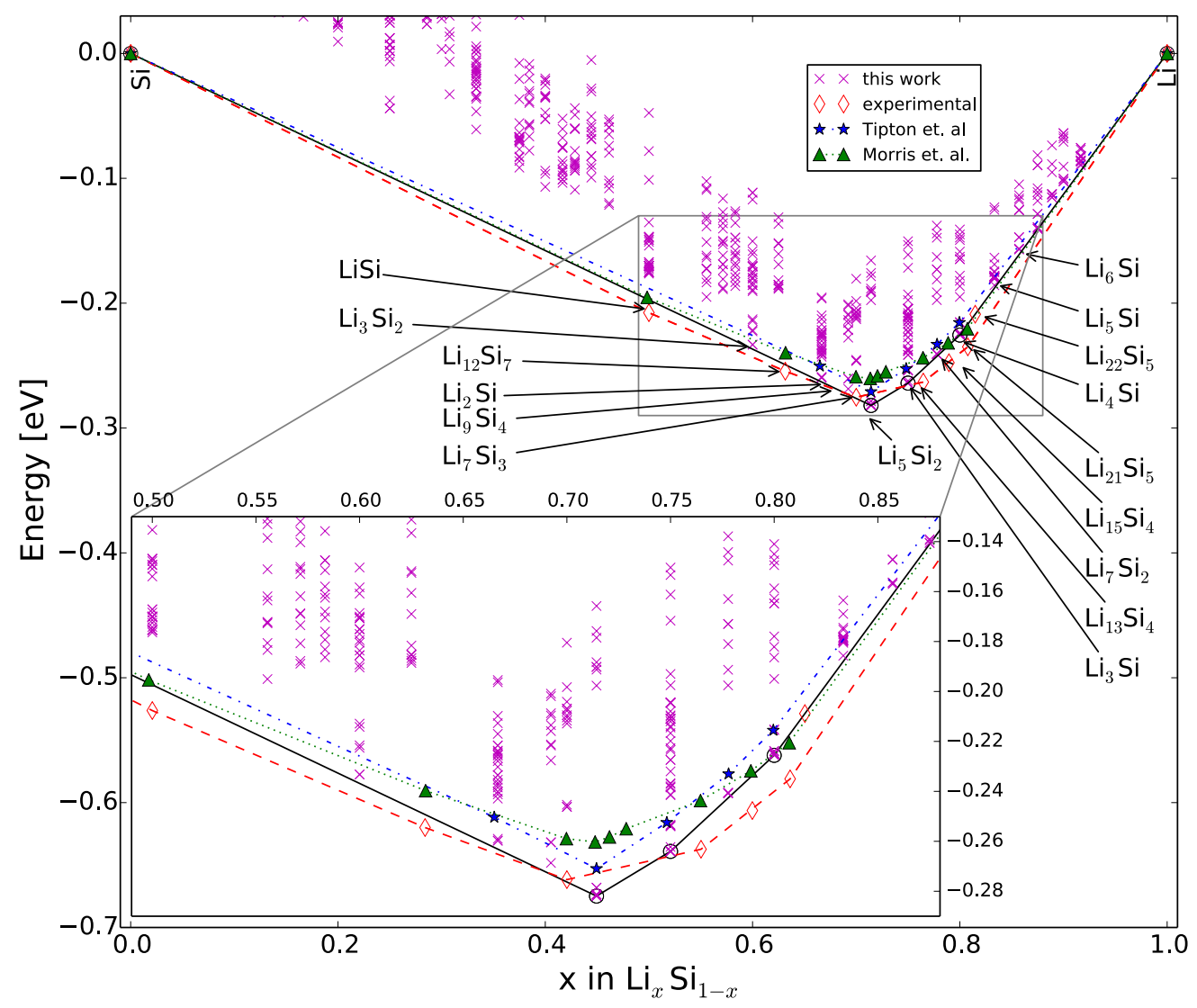

Figure 1: Convex hull of the lithium-silicon binary system. The black continuous line is obtained from $a b$ initio MHM calculations, and black circles indicate thermodynamically stables structures. The red dashed line is the convex hull constructed from the formation energy of experimental structures (red diamonds). The blue dashed-dotted line and green dotted line are the convex hulls constructed with the formation energies (blue star and green triangle symbols) of the structures reported by Morris et al. [13] and Tipton et al. [12], respectively.

the materials project [27] and OQMD [28] databases. Note that the experimental structures were also relaxed using VASP and using the same functional and convergence criteria as the ones obtained from our structural search. The (blue) dasheddotted line with (blue) stars corresponds to the predicted convex hull obtained by Tipton et. al. [12], and the (green) dotted line with (green) triangles was calculated by Morris et. al. [13]. By inspection of Fig 1, we see that our theoretical hull lies above the experimental structures of $\mathrm{Li}_{12} \mathrm{Si}_{7}, \mathrm{Li}_{7} \mathrm{Si}_{3}, \mathrm{Li}_{13} \mathrm{Si}_{4}$, $\mathrm{Li}_{22} \mathrm{Si}_{5}, \mathrm{Li}_{21} \mathrm{Si}_{5}$ and $\mathrm{Li}_{15} \mathrm{Si}_{4}$. There is an easy explanation for that: the experimentally determined crystal unit cells contain a large number of atoms compared to our maximum number of 16 atoms. More specifically, the unit cells of the above listed compositions contain 152, 60, 34, 108, 104, and 38 atoms, respectively $[29,1]$. A summary of the number of atoms per unit cell for all structures reported in this work can be found in Table 1.

We emphasize that our methodology consistently reached crystal phases at a lower energy than those previously reported by Tipton et. al. [12] and Morris et. al. [13]. These authors also used DFT to determine energies and forces, and with the same exchange-correlation functional. However, the genetic algorithm employed by Tipton et. al. is interfaced with VASP, while Morris et. al. relied on CASTEP [13] for their random search. It is not clear from where the observed discrepancies come. Even though different implementations have been used, it is expected that energy differences should be closer as compared with different calculations within the same exchange correlation functional. Therefore, we can only suggest that it could be due to different convergence criteria and the methodology used.

We do note that for the case of $\mathrm{Li}_{5} \mathrm{Si}_{2}$, one of the key results in the works of Tipton et. al. [12] and Morris et. al. [13], we find good agreement with respect to the predicted symmetry group, lattice parameters and Wyckoff positions. However, we find a small energy difference between our calculated formation energy and the ones reported by Tipton et al. [12] and Morris et al. [13], $11 \mathrm{meV}$ and $22 \mathrm{meV}$ respectively, with our structure lower in energy, a difference that could be due to the used methodologies.

Several unreported phases are characterized for the first time in our work. In fact, our MHM runs have identified two new phases for $\mathrm{Li}_{3} \mathrm{Si}$ (space group $P 12 / m 1$ ) and $\mathrm{Li}_{4} \mathrm{Si}$ (space group $I 4 / m$ ), which are on the theoretical hull and have not been previously reported. Additionally, we found novel lowenergy meta-stable structures for $\mathrm{Li}_{3} \mathrm{Si}_{2}$ (space group $C 12 / m 1$ ), $\mathrm{Li}_{2} \mathrm{Si}$ (space group $C 12 / m 1$ ), $\mathrm{Li}_{9} \mathrm{Si}_{4}$ (space group $C 12 / m 1$ ), $\mathrm{Li}_{7} \mathrm{Si}_{2}$ (space group $P \overline{3} m 1$ ), $\mathrm{Li}_{5} \mathrm{Si}$ (space group $P \overline{3} m 1$ ) and 
Table 1: All experimental and theoretical Li-Si phases studied in this work. Experimental phases from Refs. [1,29].

\begin{tabular}{lcc}
\hline Phase & space group & number of atoms \\
\hline & Experimental & \\
$\mathrm{Li}_{12} \mathrm{Si}_{7}$ & $P n m a$ & 152 \\
$\mathrm{Li}_{7} \mathrm{Si}_{3}$ & $P 3_{2} 12$ & 60 \\
$\mathrm{Li}_{13} \mathrm{Si}_{4}$ & Pbam & 34 \\
$\mathrm{Li}_{22} \mathrm{Si}_{5}$ & $F 23$ & 108 \\
$\mathrm{Li}_{21} \mathrm{Si}_{5}$ & $F \overline{4} 3 m$ & 104 \\
$\mathrm{Li}_{15} \mathrm{Si}_{4}$ & $I \overline{4} 3 d$ & 38 \\
& $T$ This work & \\
$\mathrm{Li}_{5} \mathrm{Si}_{2}$ & $R \overline{3} m$ & 7 \\
$\mathrm{Li}_{3} \mathrm{Si}$ & $P 12 / m 1$ & 12 \\
$\mathrm{Li}_{4} \mathrm{Si}_{\mathrm{Li}}$ & $I 4 / m$ & 5 \\
$\mathrm{Li}_{7} \mathrm{Si}_{2}$ & $P \overline{3} m 1$ & 9 \\
$\mathrm{Li}_{3} \mathrm{Si}_{2}$ & $C 12 / m 1$ & 10 \\
$\mathrm{Li}_{2} \mathrm{Si}$ & $C 12 / m 1$ & 12 \\
$\mathrm{Li}_{9} \mathrm{Si}$ & $C 12 / m 1$ & 13 \\
$\mathrm{Li}_{5} \mathrm{Si}$ & $P \overline{3} m 1$ & 12 \\
$\mathrm{Li}_{6} \mathrm{Si}$ & $R \overline{3} m$ & 7 \\
\hline
\end{tabular}

$\mathrm{Li}_{6} \mathrm{Si}$ (space group $R \overline{3} m$ ) which are very close to the convex hull (only 4, 3, 5, 2 and $4 \mathrm{meV} /$ atom, respectively). These energy differences are on the order of the numerical accuracy of our theoretical description. Moreover, meta-stable structures close to the convex hull could be easily stabilized experimentally by temperature, pressure, or doping. We want to stress that for some compositions we find a large set of low-energy structures close to the convex hull. This implies that for those stoichiometries the system has available many different possibilities to crystallize which could lead to the amorphization of the general structure, or the coexistence of regions of different phases, as it is also observed experimentally.

In Table 2 we summarize the crystallographic information for the crystal structures belonging to the theoretical convex hull, as well as for the most interesting meta-stable structures. The atomic arrangement that we obtained for $\mathrm{Li}_{3} \mathrm{Si}_{2}, \mathrm{Li}_{2} \mathrm{Si}, \mathrm{Li}_{9} \mathrm{Si}_{4}$, $\mathrm{Li}_{5} \mathrm{Si}_{2}, \mathrm{Li}_{3} \mathrm{Si}, \mathrm{Li}_{7} \mathrm{Si}_{2}, \mathrm{Li}_{4} \mathrm{Si}, \mathrm{Li}_{5} \mathrm{Si}$ and $\mathrm{Li}_{6} \mathrm{Si}$ are further displayed in Fig. 2. Complete crystallographic information for all structures can be found in the supplementary information. We observe a large diversity of atomic configurations. In the case of $\mathrm{Li}_{3} \mathrm{Si}_{2}$, we can see that $\mathrm{Si}$ atoms form an armchair chain, while in $\mathrm{Li}_{2} \mathrm{Si}$ they form zig-zag chains. In $\mathrm{Li}_{9} \mathrm{Si}_{4}, \mathrm{Si}$ dimers appear surrounded by $\mathrm{Li}$ atoms, and a similar arrangement is finding in $\mathrm{Li}_{5} \mathrm{Si}_{2}$. In the case of $\mathrm{Li}_{3} \mathrm{Si}$, we note the formation of $\mathrm{Si}$ dimers, but now we see isolated $\mathrm{Si}$ atoms surrounded by $\mathrm{Li}$ atoms. This bonding has similar a pattern as in the experimental structures [1, 13], where for example, we observe the formation of $\mathrm{Si}$ dimers in $\mathrm{Li}_{7} \mathrm{Si}_{3}$, and $\mathrm{Li}_{13} \mathrm{Si}_{4}$. In $\mathrm{Li}_{15} \mathrm{Si}_{4}, \mathrm{Li}_{21} \mathrm{Si}_{5}$, and $\mathrm{Li}_{22} \mathrm{Si}_{5}$ we can see the $\mathrm{Si}$ atoms become electronically isolated and surrounded by Li atoms. A similar behavior is observed in our structures.

The different arrangements can be further characterized by comparing their electron localization functions (ELF), as depicted in Fig. 3. In the case of $\mathrm{Li}_{3} \mathrm{Si}_{2}$ we can observe the lo- calization of the electrons resulting in bonding between $\mathrm{Si}-\mathrm{Si}$. But, with the increase of Li content, we see the electrons start to delocalized, increasing the metallicity. In $\mathrm{Li}_{3} \mathrm{Si}$, we can see the charge transfer between $\mathrm{Si}-\mathrm{Si}$ and isolated $\mathrm{Si}$ surrounded by $\mathrm{Li}$, resulting in electron delocalization. The same happens for the isolated $\mathrm{Si}$ atoms in $\mathrm{Li}_{4} \mathrm{Si}$. A similar observation holds for $\mathrm{Li}_{5} \mathrm{Si}$, but in this case there is also a electron delocalization between Li layers. Therefore, we can observe that with the increase of Li content the electrons are more delocalized, resulting in a metallic character of the binary compound.
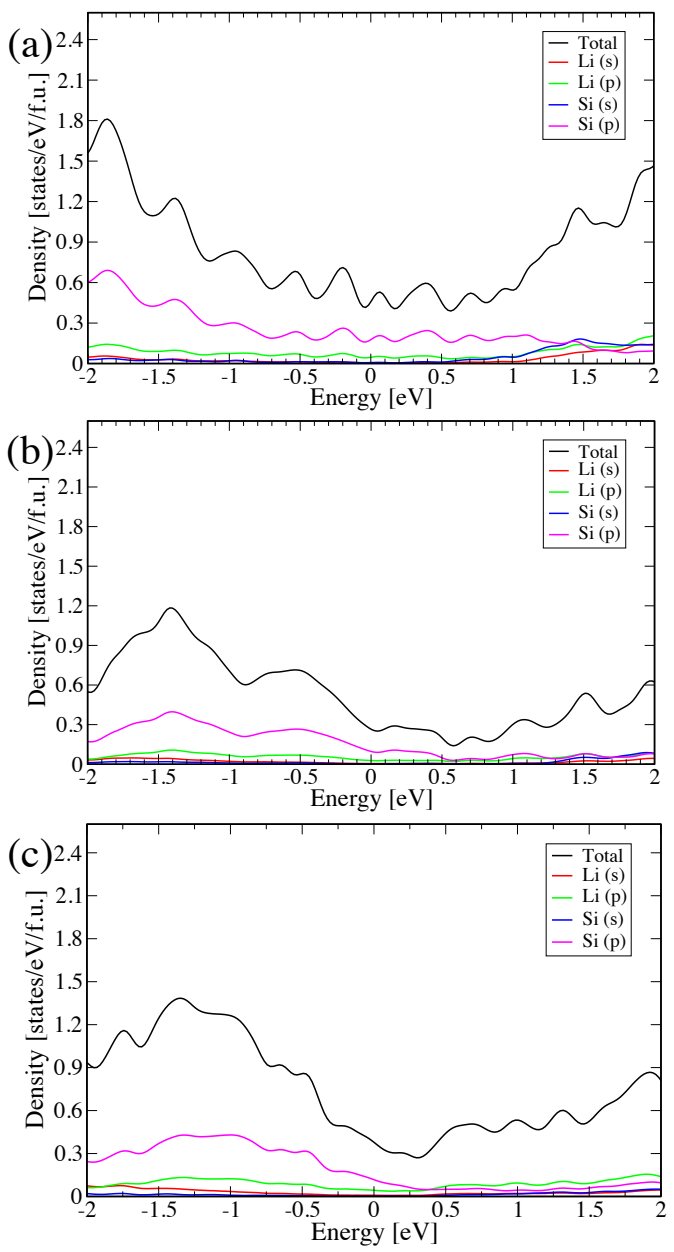

Figure 4: Total and local density of states per f.u. for (a) $\mathrm{Li}_{5} \mathrm{Si}_{2}$ (trigonal, $R \overline{3} m$ ), (b) $\mathrm{Li}_{3} \mathrm{Si}$ (monoclinic, $P 12 / m$ ), and (c) $\mathrm{Li}_{4} \mathrm{Si}$ (tetragonal, $I 4 / m$ ). Red and blue line are the $s$-orbital projections of $\mathrm{Li}$ while green and pink are the $p$-orbital projections of Si.

The Fig. 4, shows the total and local density of states (DOS) normalized per formula unit (f.u.) for the structures in the convex hull (the DOS for the meta-stable structures are reported in the supplementary information), it is clear that the structures have a predominantly metallic character, with a significant density of states at the Fermi energy. In general, we can see that most of the metallic character is coming from the $\mathrm{Si}-p$ orbitals. The exception is $\mathrm{Li}_{5} \mathrm{Si}$ and $\mathrm{Li}_{6} \mathrm{Si}$, where now the lithium content is so large that it plays a similar role as the $p$ orbitals from $\mathrm{Si}$. For the case of $\mathrm{Li}_{6} \mathrm{Si}$, the total density of states at the Fermi 

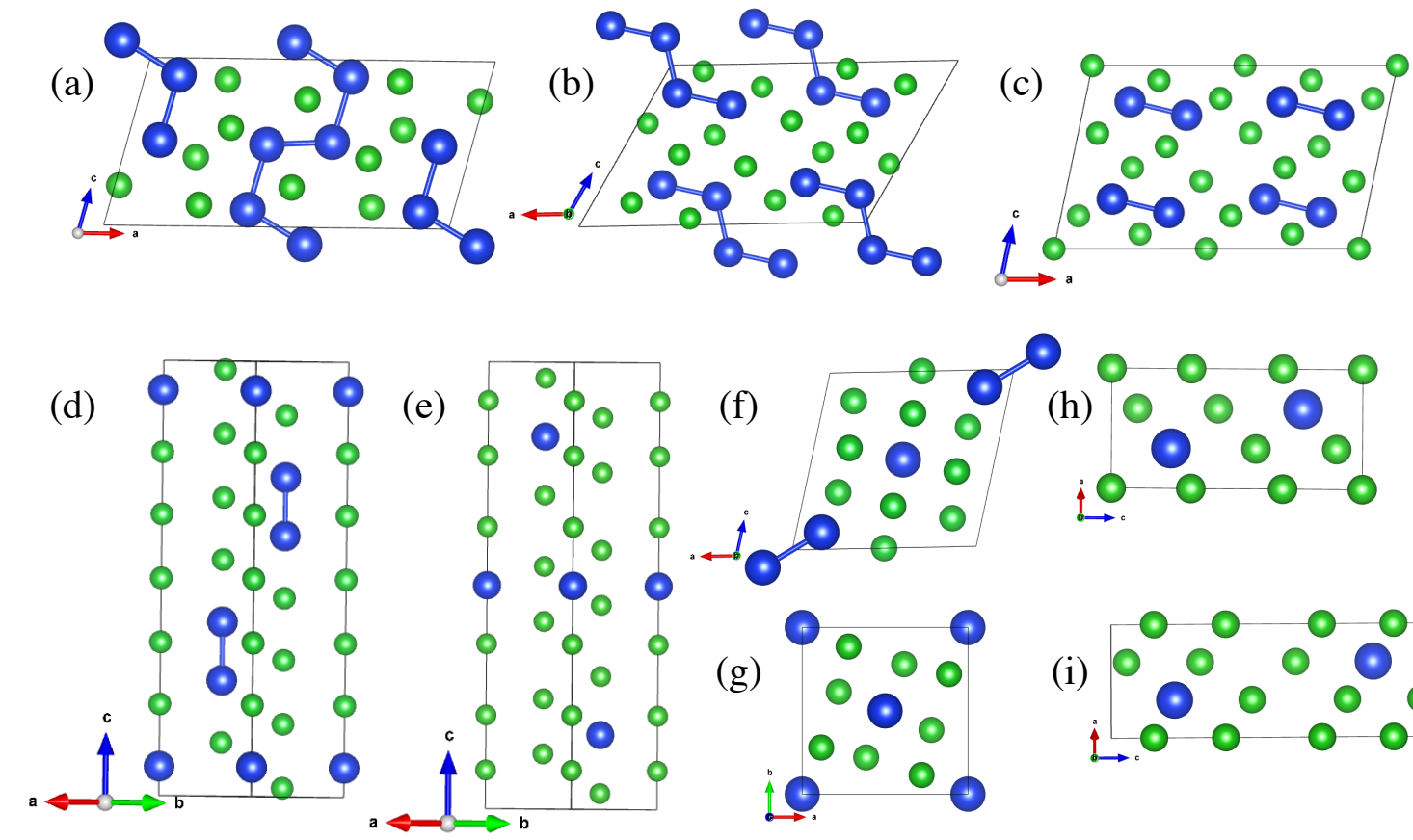

(i)

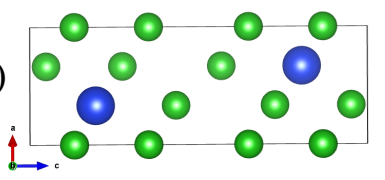

Figure 2: Crystal structure of (a) $\mathrm{Li}_{3} \mathrm{Si}_{2}$ (monoclinic, $C 12 \mathrm{~m} / 1$ ), (b) $\mathrm{Li}_{2} \mathrm{Si}$ (monoclinic, $C 12 m / 1$ ), (c) $\mathrm{Li}_{9} \mathrm{Si}_{4}$ (monoclinic, $C 12 m / 1$ ), (d) $\mathrm{Li}_{5} \mathrm{Si}_{2}$ (trigonal, $R \overline{3} m$ ) (e) $\mathrm{Li}_{6} \mathrm{Si}$ (trigonal, $R \overline{3} m$ ) (f) $\mathrm{Li}_{3} \mathrm{Si}$ (monoclinic, $P 12 / m 1$ ), (g) $\mathrm{Li}_{4} \mathrm{Si}$ (tetragonal, $I 4 / m$ ), (h) $\mathrm{Li}_{7} \mathrm{Si}_{2}$ (trigonal, $P \overline{3} m 1$ ) and (i) $\mathrm{Li}_{5} \mathrm{Si}$ (trigonal, $P \overline{3} m 1$ ). $\mathrm{Li}$ atoms are green while $\mathrm{Si}$ atoms are blue.

\begin{tabular}{|c|c|c|c|c|c|c|c|}
\hline Phase & space group & $\mathrm{a}$ & $\mathrm{b}$ & $\mathrm{c}$ & $\alpha$ & $\beta$ & $\gamma$ \\
\hline $\mathrm{Li}_{5} \mathrm{Si}_{2}$ & $R \overline{3} m$ & 4.387 & 4.387 & 17.862 & 90 & 90 & 120 \\
\hline $\mathrm{Li}_{5} \mathrm{Si}_{2}[12]$ & $R \overline{3} m$ & 4.383 & 4.383 & 17.837 & 90 & 90 & 120 \\
\hline $\mathrm{Li}_{3} \mathrm{Si}$ & $\mathrm{P} 12 / m l$ & 6.487 & 4.311 & 6.385 & 90 & 103.19 & 90 \\
\hline $\mathrm{Li}_{4} \mathrm{Si}$ & $\mathrm{I} 4 / m$ & 5.952 & 5.952 & 4.292 & 90 & 90 & 90 \\
\hline $\mathrm{Li}_{7} \mathrm{Si}_{2}$ & $P \overline{3} m 1$ & 4.375 & 4.375 & 7.958 & 90 & 90 & 120 \\
\hline $\mathrm{Li}_{3} \mathrm{Si}_{2}$ & $\mathrm{C} 12 / m 1$ & 12.217 & 4.368 & 6.147 & 90 & 75 & 90 \\
\hline $\mathrm{Li}_{2} \mathrm{Si}$ & $\mathrm{C} 12 / m 1$ & 12.232 & 4.410 & 7.937 & 90 & 90 & 120.61 \\
\hline $\mathrm{Li}_{9} \mathrm{Si}_{4}$ & $\mathrm{C} 12 / m 1$ & 12.283 & 4.38 & 7.534 & 90 & 78.12 & 90 \\
\hline $\mathrm{Li}_{5} \mathrm{Si}$ & $P \overline{3} m 1$ & 4.437 & 4.437 & 11.061 & 90 & 90 & 120 \\
\hline $\mathrm{Li}_{6} \mathrm{Si}$ & $R \overline{3} m$ & 4.448 & 4.448 & 19.995 & 90 & 90 & 120 \\
\hline
\end{tabular}

level corresponds to the largest of all considered cases, close to 1.2 states/eV/f.u. Additionally, the full band structure for each case has been included in the supplementary information.

It is well-known that the existence of imaginary frequencies in the phonon spectra indicates that the system is not dynamically stable. In Fig. 5 we present the phonon band structure of of $\mathrm{Li}_{5} \mathrm{Si}_{2}, \mathrm{Li}_{3} \mathrm{Si}$, and $\mathrm{Li}_{4} \mathrm{Si}$. The phonon band structure of the meta-stable structures are also included in the supplementary information. We clearly observe that all phonon frequencies are positive, whence unstable modes are not present. For many structures all acoustic branches have almost the same slope, indicating the isotropic elastic behavior of the material, while in a few cases large differences exist between the different branches. In order to analyze the effect of the variation of Li content on the thermodynamic properties of the materials, we report in Fig. 6 the vibrational contribution to the heat capacity. The peak on the heat capacity (normalized to $T^{3}$ ) expresses the amount of thermal energy one can acquire. Even though a certain dependence is noticed (the change in the thermal energy tends to be higher for higher lithium content), we are not able to find a clear tendency. Indeed, the maximum value is quite dependent not only on the Li content but also on the crystal structure.

We now turn our attention to the elastic properties. In the context of Li batteries, the mechanical properties are important because they are relevant for the possible anode deformation. Usually, the change in the volume during a charge and discharge process (lithiation/delithiation) can result in mechanical failure due to cracking or pulverization of the electrode $[30,31,32]$. In order to characterize the elastic behavior of these binary compounds we calculated the bulk modulus (B), shear modulus (G), Young's modulus (E), and Poisson's ratio $(v)$ for a polycrystalline material. These quantities can be obtained from the single crystal elastic stiffness constants $C_{i j}$, using the Voigt-Reuss-Hill approximation [33], which can be 


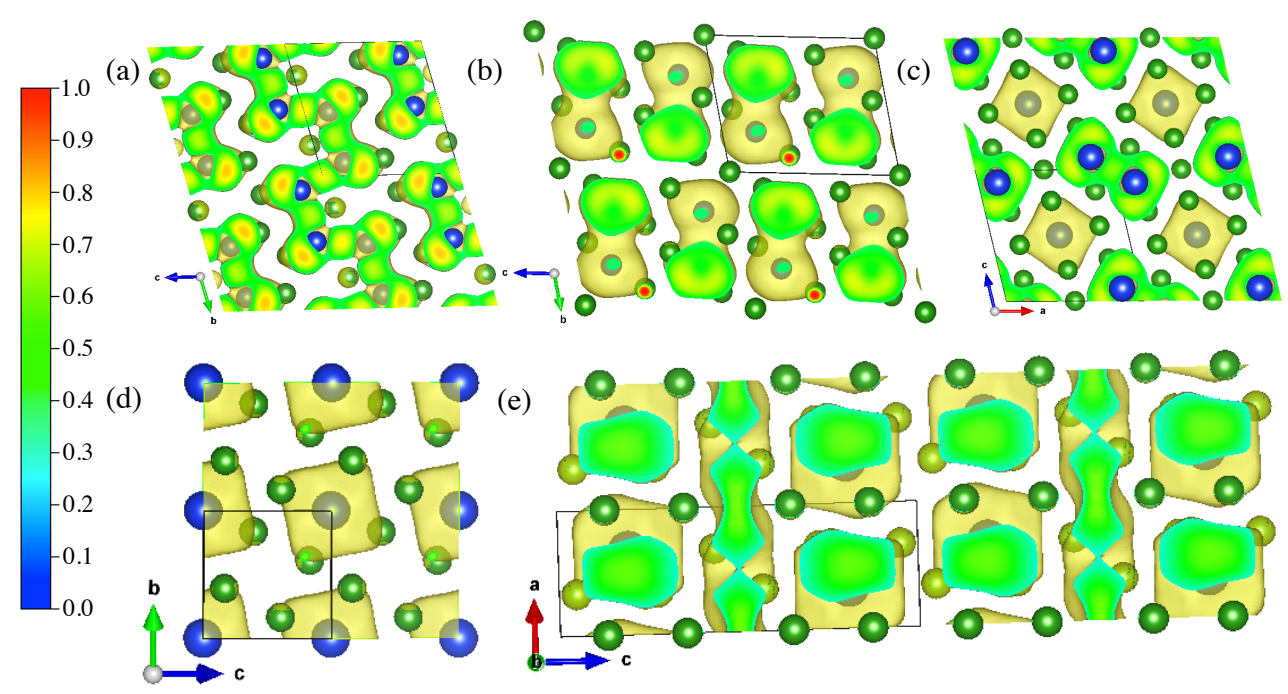

Figure 3: Electron localization function (ELF) of (a) $\mathrm{Li}_{3} \mathrm{Si}_{2}$ (monoclinic, $C 12 / m 1$ ), (b) $\mathrm{Li}_{9} \mathrm{Si}_{4}$ (monoclinic, $C 12 / m 1$ ), (c) $\mathrm{Li}_{3} \mathrm{Si}$ (monoclinic, $P 12 / m 1$ ), (d) $\mathrm{Li}_{4} \mathrm{Si}$ (tetragonal, $I 4 / m$ ), (e) $\mathrm{Li}_{5} \mathrm{Si}$ (trigonal, $P \overline{3} \mathrm{~m} 1$ ). Li atoms are green while $\mathrm{Si}$ atoms are blue. In the scale color 0.0 corresponds to localization of electrons and 1.0 corresponds to delocalization of electrons.
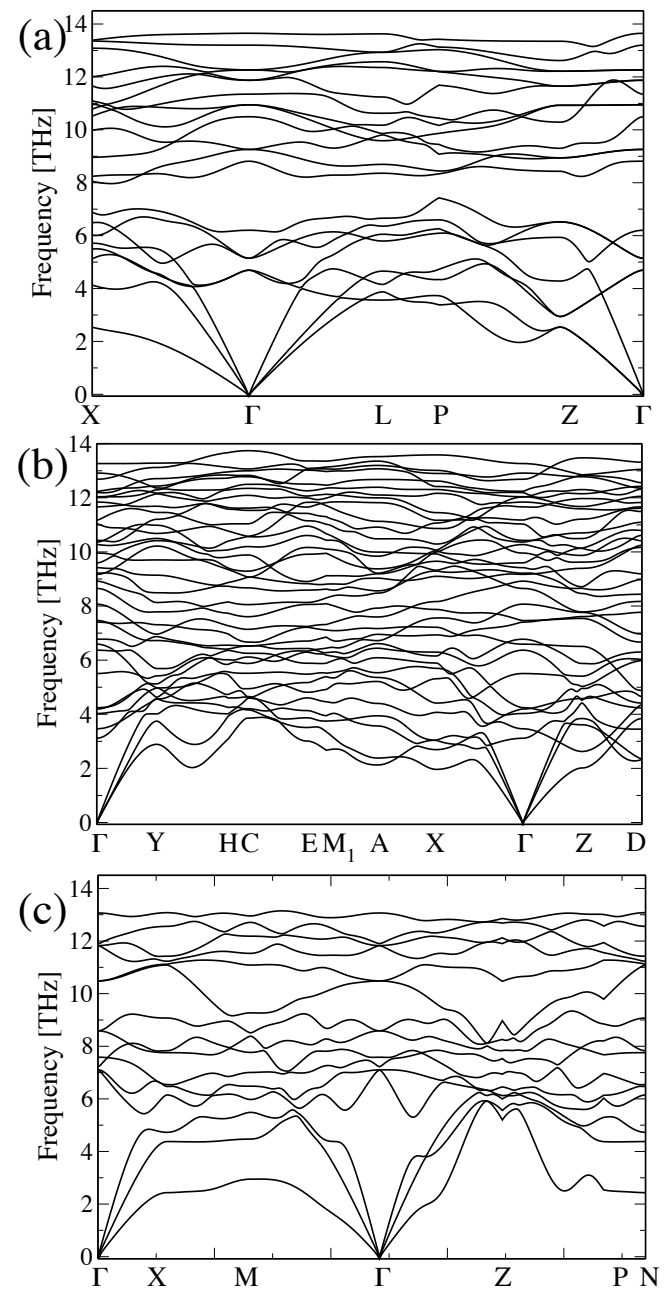

Figure 5: (a) Phonon band structures of (a) $\mathrm{Li}_{5} \mathrm{Si}_{2}(R \overline{3} m)$, (c) $\mathrm{Li}_{3} \mathrm{Si}(P 12 / m)$, and $(\mathrm{g}) \mathrm{Li}_{4} \mathrm{Si}(I 4 / m)$.

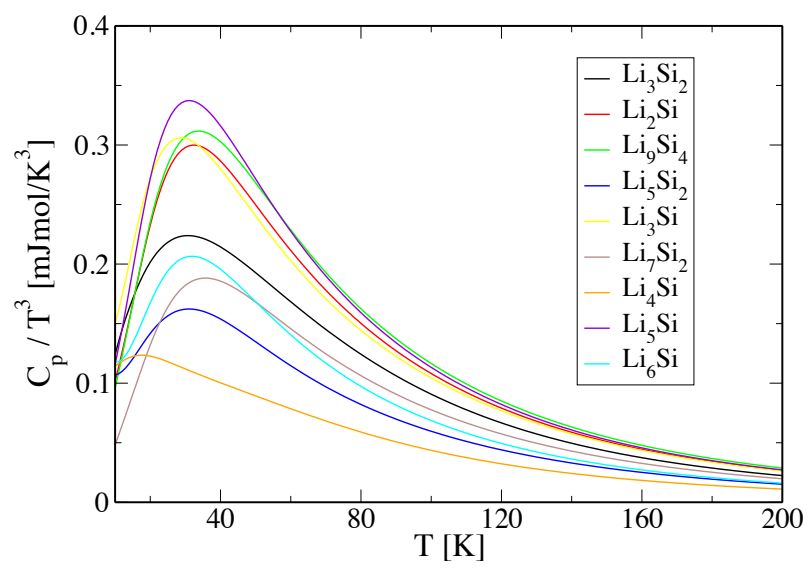

Figure 6: Vibrational contribution to the heat capacity for the lowest-energy structures reported in this work.

expressed as follows:

$$
B=\frac{1}{2}\left(B_{V}+B_{R}\right) \quad G=\frac{1}{2}\left(G_{V}+G_{R}\right)
$$

with

$$
\begin{aligned}
9 B_{V} & =\left(C_{11}+C_{22}+C_{33}\right)+2\left(C_{12}+C_{13}+C_{23}\right) \\
15 G_{V} & =\left(C_{11}+C_{22}+C_{33}+3\left(C_{44}+C_{55}+C_{66}\right)\right. \\
& -\left(C_{12}+C_{13}+C_{23}\right) \\
1 / B_{R} & =\left(s_{11}+s_{22}+s_{33}\right)+2\left(s_{12}+s_{23}+s_{31}\right) \\
15 / G_{R} & =4\left(s_{11}+s_{22}+s_{33}\right)-4\left(s_{12}+s_{23}+s_{31}\right) \\
& +3\left(s_{44}+s_{55}+s_{66}\right),
\end{aligned}
$$

where $\mathrm{C}_{i j}=1 / \mathrm{s}_{i j}$. When B and $\mathrm{G}$ have been determined, $\mathrm{E}$ and $v$ can be easily calculated using:

$$
E=\frac{9 B G}{3 B+G} \quad v=\frac{3 B-2 G}{2(3 B+G)} .
$$


Table 4: Calculated Bulk (B), shear (G), Young (E) modulus in GPa, Poisson's ratios $(v)$ and $\mathrm{B} / \mathrm{G}$ ratios.

\begin{tabular}{lccccc}
\hline Phase & $\mathrm{B}$ & $\mathrm{G}$ & $\mathrm{E}$ & $v$ & $\mathrm{~B} / \mathrm{G}$ \\
\hline $\mathrm{Si}$ & 89.5 & 62.7 & 152.9 & 0.22 & 1.4 \\
$\mathrm{Si}$ exp [9] & 97.6 & & & & \\
$\mathrm{Si} \mathrm{ref[5]}$ & 88.32 & 64.91 & 156.13 & 0.19 & 1.4 \\
$\mathrm{Li}_{3} \mathrm{Si}_{2}$ & 38.4 & 29.1 & 69.7 & 0.19 & 1.3 \\
$\mathrm{Li}_{2} \mathrm{Si}_{\mathrm{Li}}$ & 37.8 & 29.1 & 69.4 & 0.19 & 1.3 \\
$\mathrm{Si}_{4}$ & 37.1 & 31.2 & 73.0 & 0.17 & 1.2 \\
$\mathrm{Li}_{5} \mathrm{Si}_{2}$ & 39.8 & 34.8 & 79.8 & 0.16 & 1.1 \\
$\mathrm{Li}_{3} \mathrm{Si}$ & 35.9 & 30.7 & 71.2 & 0.17 & 1.2 \\
$\mathrm{Li}_{7} \mathrm{Si}$ & 33.9 & 27.5 & 64.7 & 0.18 & 1.2 \\
$\mathrm{Li}_{4} \mathrm{Si}$ & 29.7 & 26.6 & 60.7 & 0.16 & 1.1 \\
$\mathrm{Li}{ }_{5} \mathrm{Si}$ & 26.5 & 18.0 & 43.3 & 0.22 & 1.5 \\
$\mathrm{Li}$ & 23.9 & 16.1 & 39.0 & 0.22 & 1.5 \\
$\mathrm{Li}$ & 14.1 & 6.7 & 17.5 & 0.3 & 2.1 \\
$\mathrm{Li} \exp [38]$ & 12.13 & 8.78 & 21.22 & 0.21 & 1.38 \\
$\mathrm{Li}$ ref[5] & 13.61 & 10.01 & 37.96 & 0.14 & 1.35 \\
\hline
\end{tabular}

Table 3 presents the elastic constants of all phases here reported. Likewise, in Table 4 we summarize the bulk, shear, and Young modulus as well as the Poisson's ratio and the $\mathrm{B} / \mathrm{G}$ ratio for the phases reported and for the pure elements ( $\mathrm{Li}$ and $\mathrm{Si}$ ). It is well known that the ration $B / G$ can be used to classify brittle and ductile behavior in materials $[34,35]$. We can observe that the bulk modulus decrease monotonically with the increase of the Li content, while Young's Modulus is constant up to $71 \%$ of Li, before beginning to decrease. $B / G$ ratios smaller than 1.75 are associated with brittle alloys, while values larger than 1.75 with ductile materials. Looking at our structures, we can see that $B / G$ is smaller than 1.75 , suggesting that all structures are brittle. This observation is in agreement with those reported by J. Xia et. al. [36]. With the increase of Li content, the presence of covalent bonds decreases and the presence of metallic bonds increases due to the electron delocalization, making the whole structure weaker. A further confirmation comes from the determination of the Cauchy pressure, which is estimated from the difference between $\mathrm{C}_{12}$ and $\mathrm{C}_{44}$ (if it is negative, the crystal tends to be brittle). The phase having highest Li content in this work is $\mathrm{Li}_{6} \mathrm{Si}$ and its bulk modulus is smaller than the value for Si by almost one order of magnitude. This behavior clearly suggests an elastic softening and mechanical failure in phases with high Li content and an overall strong dependency of the mechanical properties on $\mathrm{Li}$ concentration. We note that our results are in qualitative agreement with those related to experimental phases [5, 37, 32].

Figure 7 shows the electric potential profile as a function of Li content for the structures belonging to our theoretical convex hull. The voltages were calculated as previously described in section 2. We also compare our calculated potential profile with the experimental one reported at $415^{\circ} \mathrm{C}$ by Wen et al [6]. We can see a similar behavior in the electric potential as a function of the Li content using our structures, despite the fact that we do not include temperature effects. Our electric potential profile is in agreement with those experimentally and theoreti-

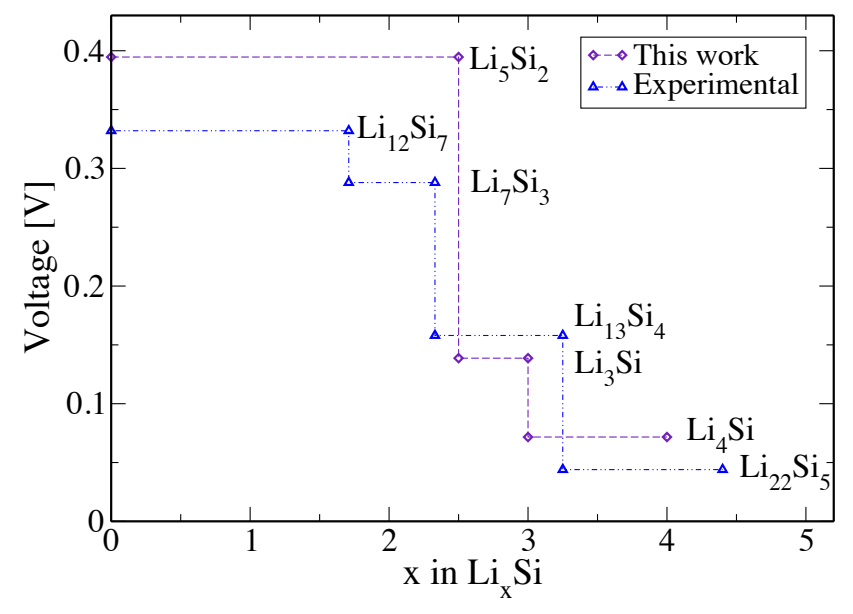

Figure 7: Electric potential profile of Li-Si alloys as a function of the Li content compared with the profile calculated using experimental structures.

cally reported $[12,13,1]$.

\section{Summary}

In summary, we report a set of new crystal structures for LiSi binary compounds obtained by using the minima hopping method, and by varying the chemical composition. In order to understand the electronic properties of these compounds, we have calculated the density of states and electron localization functions. We find that the structures are mostly metallic, with metallicity increasing with the lithium content. Additionally, we verified the dynamical stability of all considered structures by calculating the phonon spectra. We further characterized the elastic properties by determining the bulk modulus, shear modulus, Young modulus, and Poisson's ratio. We observed that mechanical properties, phonon dispersion, and electron localization functions are strongly sensitive to the Li content and depend on the nature of the bonds. We finally calculate the electric profile as a function of the Li content, finding an overall agreement with data in literature.

\section{Acknowledgments}

This work used the Extreme Science and Engineering Discovery Environment (XSEDE), which is supported by National Science Foundation grant number OCI-1053575. Additionally, the authors acknowledge the support from the Texas Advanced Computer Center (TACC) and Super Computing System (Mountaineer) at WVU, which are funded in part by the National Science Foundation EPSCoR Research Infrastructure Improvement Cooperative Agreement 1003907, the state of West Virginia (WVEPSCoR via the Higher Education Policy Commission) and WVU. We thank Dr. Guillermo Avedaño Franco for fruitful discussions. A.H. Romero and I. ValenciaJaime acknowledge the support of the Marie Curie Actions from the European Union in the international incoming fellowships (grant PIIFR-GA-2011-911070), project CONACYTMéxico 152153 and the Donors of the American Chemical 
Table 3: Computed elastic constants for Li-Si binaries here reported. All quantities are in units of GPa.

\begin{tabular}{rrrrrrrrrr}
\hline & $\mathrm{Li}_{3} \mathrm{Si}_{2}$ & $\mathrm{Li}_{2} \mathrm{Si}$ & $\mathrm{Li}_{9} \mathrm{Si}_{4}$ & $\mathrm{Li}_{5} \mathrm{Si}_{2}$ & $\mathrm{Li}_{3} \mathrm{Si}$ & $\mathrm{Li}_{7} \mathrm{Si}_{2}$ & $\mathrm{Li}_{4} \mathrm{Si}$ & $\mathrm{Li}_{5} \mathrm{Si}_{1} \mathrm{Li}_{6} \mathrm{Si}$ \\
\hline $\mathrm{C}_{11}$ & 125.3 & 110.0 & 124.6 & 102.1 & 78.4 & 85.9 & 49.0 & 73.4 & 61.7 \\
$\mathrm{C}_{12}$ & 13.9 & 2.4 & 3.8 & 16.6 & -2.7 & 17.9 & 24.1 & 10.2 & 7.1 \\
$\mathrm{C}_{13}$ & 12.3 & 19.1 & 1.7 & -9.7 & 33.8 & 3.0 & 13.0 & 2.5 & 8.7 \\
$\mathrm{C}_{14}$ & & & & -15.6 & & -12.2 & & 11.9 & -10.7 \\
$\mathrm{C}_{15}$ & 0.7 & -15.8 & -1.6 & & -5.4 & & & & \\
$\mathrm{C}_{16}$ & & & & & & & -20.9 & & \\
$\mathrm{C}_{22}$ & 85.8 & 82.1 & 85.5 & 102.1 & 89.5 & 85.9 & 49.1 & 73.4 & 61.7 \\
$\mathrm{C}_{23}$ & 3.4 & 19.4 & 17.0 & -9.7 & 22.7 & 3.0 & 13.0 & 2.5 & 8.7 \\
$\mathrm{C}_{25}$ & -0.8 & -5.8 & -6.9 & & 1.1 & & & & \\
$\mathrm{C}_{33}$ & 84.1 & 69.7 & 81.4 & 162.1 & 49.3 & 86.5 & 70.4 & 63.1 & 43.6 \\
$\mathrm{C}_{35}$ & -1.1 & 12.7 & 6.1 & & -4.9 & & & & \\
$\mathrm{C}_{44}$ & 14.9 & 35.1 & 30.1 & 24.0 & 48.5 & 22.1 & 32.9 & 11.9 & 12.4 \\
$\mathrm{C}_{46}$ & 2.5 & -1.9 & -6.4 & & -2.7 & & & & \\
$\mathrm{C}_{55}$ & 33.1 & 36.1 & 23.2 & 24.0 & 58.7 & 22.1 & 32.9 & 11.9 & 12.6 \\
$\mathrm{C}_{66}$ & 22.9 & 16.4 & 24.6 & 42.8 & 21.9 & 33.9 & 36.1 & 27.3 & 27.3 \\
\hline
\end{tabular}

Society Petroleum Research Fund for partial support of this research under contract 54075-ND10. M.A.L.M. and S. B. acknowledge financial support from the French ANR project ANR-12-BS04-0001-02.

\section{References}

[1] V. Chevrier, J. Zwanziger, J. Dahn, First principles study of Li-Si crystalline phases: Charge transfer, electronic structure, and lattice vibrations, Journal of Alloys and Compounds 496 (2010) 25 - 36.

[2] C. K. Chan, H. Peng, G. Liu, K. McIlwrath, X. F. Zhang, R. a. Huggins, Y. Cui, High-performance lithium battery anodes using silicon nanowires., Nature nanotechnology 3 (2008) 31-5.

[3] L. Y. Beaulieu, T. D. Hatchard, A. Bonakdarpour, M. D. Fleischauer, J. R. Dahn, Reaction of Li with alloy thin films studied by in situ AFM, Journal of The Electrochemical Society 150 (2003) A1457-A1464.

[4] W. Wang, P. N. Kumta, Reversible high capacity nanocomposite anodes of Si/C/SWNTs for rechargeable Li-ion batteries, Journal of Power Sources 172 (2007) $650-658$.

[5] V. Shenoy, P. Johari, Y. Qi, Elastic softening of amorphous and crystalline Li-Si phases with increasing Li concentration: A first-principles study, Journal of Power Sources 195 (2010) 6825 - 6830.

[6] C. J. Wen, R. A. Huggins, Chemical diffusion in intermediate phases in the Lithium-Silicon system, Journal of Solid State Chemistry 37 (1981) $271-278$.

[7] H. Okamoto, Li-Si (Lithium-Silicon), Journal of Phase Equilibria and Diffusion 30 (2009) 118-119.

[8] R. Nesper, H. G. von Schnering, $\mathrm{Li}_{21} \mathrm{Si}_{5}$, a zintl phase as well as a humerothery phase, Journal of Solid State Chemistry 70 (1987) 48 - 57.

[9] Z. Zeng, N. Liu, Q. Zeng, Y. Ding, S. Qu, Y. Cui, W. L. Mao, Elastic moduli of polycrystalline $\mathrm{Li}_{15} \mathrm{Si}_{4}$ produced in Lithium ion batteries, Journal of Power Sources 242 (2013) 732 - 735.

[10] J. Li, J. R. Dahn, An in situ X-Ray diffraction study of the reaction of Li with crystalline Si, Journal of The Electrochemical Society 154 (2007) A156-A161.

[11] X. H. Liu, L. Q. Zhang, L. Zhong, Y. Liu, H. Zheng, J. W. Wang, J.H. Cho, S. A. Dayeh, S. T. Picraux, J. P. Sullivan, S. X. Mao, Z. Z. Ye, J. Y. Huang, Ultrafast electrochemical lithiation of individual Si nanowire anodes, Nano Letters 11 (2011) 2251-2258.

[12] W. W. Tipton, C. R. Bealing, K. Mathew, R. G. Hennig, Structures, phase stabilities, and electrical potentials of Li-Si battery anode materials, Phys. Rev. B 87 (2013) 184114-1-184114-7.

[13] A. J. Morris, C. P. Grey, C. J. Pickard, Thermodynamically stable lithium silicides and germanides from density functional theory calculations, Phys. Rev. B 90 (2014) 054111-1-054111-9.
[14] M. Zeilinger, D. Benson, U. Hussermann, T. F. Fssler, Single crystal growth and thermodynamic stability of $\mathrm{Li}_{17} \mathrm{Si}_{4}$, Chemistry of Materials 25 (2013) 1960-1967.

[15] S. Goedecker, Minima hopping: An efficient search method for the global minimum of the potential energy surface of complex molecular systems, The Journal of Chemical Physics 120 (2004) 9911-9917.

[16] M. Amsler, S. Goedecker, Crystal structure prediction using the minima hopping method, The Journal of Chemical Physics 133 (2010) 224104 1-224104-8.

[17] G. Kresse, J. Furthmüller, Efficient iterative schemes for ab initio totalenergy calculations using a plane-wave basis set, Phys. Rev. B 54 (1996) 11169-11186.

[18] G. Kresse, D. Joubert, From ultrasoft pseudopotentials to the projector augmented-wave method, Phys. Rev. B 59 (1999) 1758-1775.

[19] P. E. Blöchl, Projector augmented-wave method, Phys. Rev. B 50 (1994) 17953-17979.

[20] J. P. Perdew, K. Burke, M. Ernzerhof, Generalized gradient approximation made simple, Phys. Rev. Lett. 77 (1996) 3865-3868.

[21] H. J. Monkhorst, J. D. Pack, Special points for brillouin-zone integrations, Phys. Rev. B 13 (1976) 5188-5192.

[22] A. Togo, F. Oba, I. Tanaka, First-principles calculations of the ferroelastic transition between rutile-type and $\mathrm{CaCl} 2$-type $\mathrm{SiO}_{2}$ at high pressures, Phys. rev. B 78 (2008) 134106-1-134106-8.

[23] Q. Zhang, Y. Cui, E. Wang, First-principles approaches to simulate lithiation in silicon electrodes, Modelling and Simulation in Materials Science and Engineering 21 (2013) 074001-1-074001-29.

[24] I. A. Courtney, J. S. Tse, O. Mao, J. Hafner, J. R. Dahn, Ab initio calculation of the lithium-tin voltage profile, Phys. Rev. B 58 (1998) 1558315588.

[25] M. K. Aydinol, A. F. Kohan, G. Ceder, K. Cho, J. Joannopoulos, Ab initio study of lithium intercalation in metal oxides and metal dichalcogenides, Phys. Rev. B 56 (1997) 1354-1365.

[26] M. K. Aydinol, G. Ceder, Firstprinciples prediction of insertion potentials in Li-Mn oxides for secondary Li batteries, Journal of The Electrochemical Society 144 (1997) 3832-3835.

[27] A. Jain, S. P. Ong, G. Hautier, W. Chen, W. D. Richards, S. Dacek, S. Cholia, D. Gunter, D. Skinner, G. Ceder, K. a. Persson, Commentary: The Materials Project: A materials genome approach to accelerating materials innovation, APL Materials 1 (2013) 011002-1-011002-11.

[28] J. Saal, S. Kirklin, M. Aykol, B. Meredig, C. Wolverton, Materials Design and Discovery with High-Throughput Density Functional Theory: The Open Quantum Materials Database (OQMD), JOM 65 (2013) 15011509.

[29] V. L. Chevrier, J. W. Zwanziger, J. R. Dahn, First principles studies of silicon as a negative electrode material for Lithium-ion batteries, Canadian Journal of Physics 87 (2009) 625-632.

[30] M. J. Chon, V. A. Sethuraman, A. McCormick, V. Srinivasan, P. R. 
Guduru, Real-time measurement of stress and damage evolution during initial lithiation of crystalline silicon, Phys. Rev. Lett. 107 (2011) 045503-1-045503-4.

[31] C.-Y. Chou, H. Kim, G. S. Hwang, A comparative first-principles study of the structure, energetics, and properties of $\mathrm{Li}-\mathrm{M}(\mathrm{M}=\mathrm{Si}, \mathrm{Ge}, \mathrm{Sn})$ Alloys, The Journal of Physical Chemistry C 115 (2011) 20018-20026.

[32] K. Li, H. Xie, J. Liu, Z. Ma, Y. Zhou, D. Xue, From chemistry to mechanics: bulk modulus evolution of $\mathrm{Li}-\mathrm{Si}$ and $\mathrm{Li}-\mathrm{Sn}$ alloys via the metallic electronegativity scale, Phys. Chem. Chem. Phys. 15 (2013) 1765817663.

[33] R. Hill, The elastic behaviour of a crystalline aggregate, Proceedings of the Physical Society. Section A 65 (1952) 349-352.

[34] S. Pugh, XCII. Relations between the elastic moduli and the plastic properties of polycrystalline pure metals, Philosophical Magazine Series 745 (1954) 823-843.

[35] S. Boucetta, Theoretical study of elastic, mechanical and thermodynamic properties of $\mathrm{MgRh}$ intermetallic compound, Journal of Magnesium and Alloys 2 (2014) $59-63$.

[36] J. Xia, E. A. Carter, Orbital-free density functional theory study of crystalline Li-Si alloys, Journal of Power Sources 254 (2014) 62 - 72.

[37] J. Moon, K. Cho, M. Cho, Ab-initio study of silicon and tin as a negative electrode materials for Lithium-ion batteries, International Journal of Precision Engineering and Manufacturing 13 (2012) 1191-1197.

[38] J. Trivisonno, C. S. Smith, Elastic constants of Lithium-Magnesium alloys, Acta Metallurgica 9 (1961) $1064-1071$. 